\title{
Free vibrations of a Reddy-Bickford multi-span beam carrying multiple spring-mass systems
}

\author{
Yusuf Yesilce* \\ Dokuz Eylul University, Civil Engineering Department, Eng. Fac., 35160, Buca, Izmir, Turkey
}

Received 21 August 2009

Revised 4 May 2010

Abstract. The structural elements supporting motors or engines are frequently seen in technological applications. The operation of machine may introduce additional dynamic stresses on the beam. It is important, then, to know the natural frequencies of the coupled beam-mass system, in order to obtain a proper design of the structural elements. The literature regarding the free vibration analysis of Bernoulli-Euler and Timoshenko single-span beams carrying a number of spring-mass system and multi-span beams carrying multiple spring-mass systems are plenty, but the free vibration analysis of Reddy-Bickford multi-span beams carrying multiple spring-mass systems has not been investigated by any of the studies in open literature so far. This paper aims at determining the exact solutions for the natural frequencies and mode shapes of Reddy-Bickford beams. The model allows analyzing the influence of the shear effect and spring-mass systems on the dynamic behavior of the beams by using Reddy-Bickford Beam Theory (RBT). The effects of attached spring-mass systems on the free vibration characteristics of the 1-4 span beams are studied. The natural frequencies of Reddy-Bickford single-span and multi-span beams calculated by using the numerical assembly technique and the secant method are compared with the natural frequencies of single-span and multi-span beams calculated by using Timoshenko Beam Theory (TBT); the mode shapes are presented in graphs.

Keywords: Eigenvalue problem, free vibration, numerical assembly technique, Reddy-Bickford multi-span beam, spring-mass system

\section{Introduction}

The analysis of beams has been performed over the years mostly using Bernoulli-Euler beam theory (BET). The classical Bernoulli-Euler beam is well studied for slender beams, where the transverse shear deformation can be safely disregarded. This theory is based on the assumption that plain sections of the cross-section remain plain and perpendicular to the beam axis. The cross-sectional displacements of Bernoulli-Euler beam theory are shown in (Fig. 1.a) [1]. For moderately thick beams Bernoulli-Euler beam theory can be modified in order to take into account the transverse shear effect in a simplified way. For example, the well-known Timoshenko beam theory (TBT) predicts a uniform shear distribution, so necessitating the use of a so-called shear factor [2-5]. The cross-sectional displacements of Timoshenko beam theory are shown in (Fig. 1.b). Han et al. presented a comprehensive study of Bernoulli-Euler, Rayleigh, Shear and Timoshenko beam theories [6].

The real shear deformation distribution is not uniform along the depth of the beam, so that Timoshenko beam theory is not recommended for composite beams, where the accurate determination of the shear stresses is required. Especially, it was found that the Timoshenko shear deformation theory has some major numerical problems such as

${ }^{*}$ Corresponding author. Tel.: +90 232 4127073; Fax: +90 232 4531191; E-mail: yusuf.yesilce@ deu.edu.tr. 


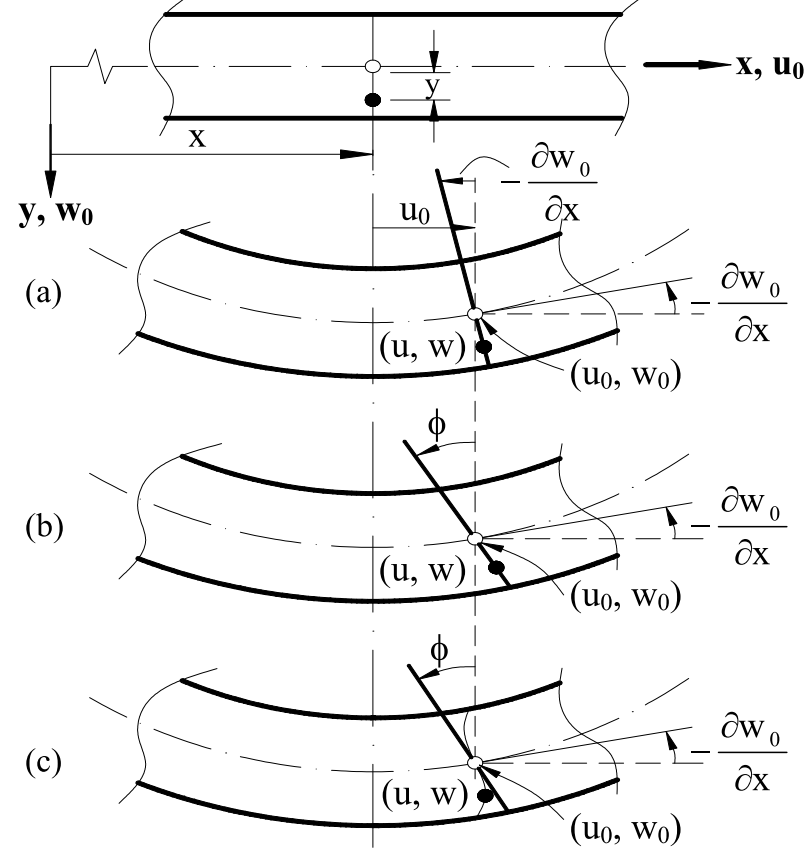

Fig. 1. Cross-section displacements in different beam theories [10]. a. Bernoulli-Euler Beam Theory (BET); b. Timoshenko Beam Theory (TBT); c. Reddy-Bickford Beam Theory (RBT).

locking in the numerical analysis for composite materials. The other problem was the need to supply an artificially derived shear correction factor. Although some remedies were devised, as a result, several higher-order theories have emerged. These theories, with small variations, are due to Bickford, Levinson, Heyliger and Reddy, Wang et al. and others all relax the restriction on the warping of the cross-section and allow variation in the longitudinal direction of the beam which is cubic [7-10]. In this paper, Reddy-Bickford beam theory (RBT) is used, which seems a good compromise between accuracy and simplicity $[7,10]$. The cross-sectional displacements of Reddy-Bickford beam theory are shown in (Fig. 1.c).

Bernoulli-Euler beam theory does not consider the shear stress in the cross-section and the associated strains. Thus, the shear angle is taken as zero through the height of the cross-section. Timoshenko beam theory assumes constant shear stress and shear strain in the cross-section. On the top and bottom edges of the beam the free surface condition is thus violated. The use of a shear correction factor, in various forms including the effect of Poisson's ratio, does not correct this fault of the theory, but rather artificially adjusts the solutions to match the static or dynamic behavior of the beam. Reddy-Bickford beam theory and the other high-order theories remedy this physical mismatch at the free edges by assuming variable shear strain and shear stress along the height of the cross-section. Then there is no need for the shear correction factor. The high-order theory is more exact and represents much better the physics of the problem. It results in a sixth-order theory compared to the fourth order of the other less-accurate theories. This yields a six-degree-of-freedom element with six end forces, a shear force, bending moment and a high-order moment, at the two ends of the beam element.

Extensive research has been carried out with regard to the vibration analysis of beams carrying concentrated masses at arbitrary positions and additional complexities.

Introducing the mass by the Dirac delta function, Chen solved analytically the problem of a simply supported beam carrying a concentrated mass [11]. Chang solved a simply supported Rayleigh beam carrying a rigidly attached centered mass [12]. Dowell studied general properties of beams carrying springs and concentrated masses [13]. Gürgöze used the normal mode summation technique to determine the fundamental frequency of the cantilever beams carrying masses and springs [14,15]. Gürgöze investigated the eigenfrequencies of a cantilever beam with attached tip mass and a spring-mass system [16]. In the other study, the alternative formulations of the frequency equation of a Bernoulli-Euler beam to which several spring-mass systems are attached in-span were investigated by 
Gürgöze [17]. Lin and Tsai investigated the free vibration analysis of a uniform Bernoulli-Euler multi-span beam carrying multiple point masses and Bernoulli-Euler multiple-step beam carrying a number of intermediate lumped masses and rotary inertias $[18,19]$. In the other study, Lin and Tsai determined the natural frequencies and mode shapes of Bernoulli-Euler multi-span beam carrying multiple spring-mass systems [20]. Wu determined the natural frequencies and mode shapes of a uniform Bernoulli-Euler cantilever beam carrying any number of spring-mass systems by using the conventional finite element method and the equivalent mass method [21]. Liu et al. formulated the frequency equation for beams carrying intermediate concentrated masses by using the Laplace Transformation Technique [22]. Wu and Chou obtained the exact solution of the natural frequency values and mode shapes for a beam carrying any number of spring masses [23]. Naguleswaran obtained the natural frequency values of the beams on up to five resilient supports including ends and carrying several particles by using BET obtained a fourth-order determinant equated to zero [24,25]. Zhou studied the free vibration of multi-span Timoshenko beams by using Rayleigh-Ritz method [26]. He developed the static Timoshenko beam functions which are composed of a set of transverse deflection functions and a set of rotational angle functions as the trial functions. Wu and Chen performed the free vibration analysis of a uniform Timoshenko beam carrying multiple spring-mass systems by using numerical assembly method [27]. Lin and Chang studied the free vibration analysis of a multi-span Timoshenko beam with an arbitrary number of flexible constraints by considering the compatibility requirements on each constraint point and using a transfer matrix method [28]. Wang et al. studied the natural frequencies and mode shapes of a uniform Timoshenko beam carrying multiple intermediate spring-mass systems with the effects of shear deformation and rotatory inertia [29]. Yesilce and Demirdag investigated the effect of axial force on free vibration of Timoshenko multi-span beam carrying multiple spring-mass systems [30]. Other studies on the vibration analysis of beams carrying masses are presented in references [31,32].

\section{The mathematical model and formulation}

A uniform Reddy-Bickford beam supported by $T$ pins included at the two ends of beam and carrying $S$ springmass systems is presented in (Fig. 2). From (Fig. 2), the total number of stations is $N=S+T[20,30]$. The kinds of coordinates which are used in this study are given below:

$x_{v^{\prime}}$ are the position coordinates for the stations, $\left(1 \leqslant v^{\prime} \leqslant N\right)$,

$x_{p}^{*}$ are the position coordinates of the spring-mass systems, $(1 \leqslant p \leqslant S)$,

$\bar{x}_{r}$ are the position coordinates of the pinned supports, $(1 \leqslant r \leqslant T)$.

From (Fig. 2), the symbols of $1^{\prime}, 2^{\prime}, \ldots, \mathbf{v}^{\prime}, \ldots, \mathbf{N}^{\prime}-1, \mathbf{N}^{\prime}$ above the $x$-axis refer to the numbering of stations. The symbols of $1,2, \ldots, \mathrm{p}, \ldots, \mathrm{S}$ below the $x$-axis refer to the numbering of spring-mass systems. The symbols of (1), (2), .., (r), .., T below the $x$-axis refer to the numbering of pinned supports.

According to Reddy-Bickford beam theory, the displacements can be written as [10]:

$$
\begin{aligned}
& u(x, y, t)=y \cdot \phi(x, t)-\alpha \cdot y^{3} \cdot\left[\phi(x, t)+\frac{\partial w(x, t)}{\partial x}\right] \\
& w(x, y, t)=w_{0}(x, t)
\end{aligned}
$$

where $w_{0}(x, t)$ is the lateral displacement of the beam neutral axis; $\phi(x, t)$ represents the rotation of a normal to the axis of the beam; $u(x, y, t)$ and $w(x, y, t)$ are the axial and lateral displacements of the beam, respectively; $x$ is the beam position; $y$ is the distance from the beam neutral axis; $t$ is time variable; $h$ is the height of the beam and $\alpha=\frac{4}{3 \cdot h^{2}}$.

Using Hamilton's principle, Eqs (1) and (2); the equations of motion can be written as:

$$
\begin{aligned}
& -\frac{68}{105} \cdot E I_{x} \cdot \frac{\partial^{2} \phi(x, t)}{\partial x^{2}}+\frac{16}{105} \cdot E I_{x} \cdot \frac{\partial^{3} w(x, t)}{\partial x^{3}}+\frac{8}{15} \cdot A G \cdot\left[\phi(x, t)+\frac{\partial w(x, t)}{\partial x}\right]=0 \\
& -m \cdot \frac{\partial^{2} w(x, t)}{\partial t^{2}}+\frac{8}{15} \cdot A G \cdot\left[\frac{\partial \phi(x, t)}{\partial x}+\frac{\partial^{2} w(x, t)}{\partial x^{2}}\right]+\frac{16}{105} \cdot E I_{x} \cdot \frac{\partial^{3} \phi(x, t)}{\partial x^{3}}-\frac{1}{21} \cdot E I_{x} \cdot \frac{\partial^{4} w(x, t)}{\partial x^{4}}=0
\end{aligned}
$$




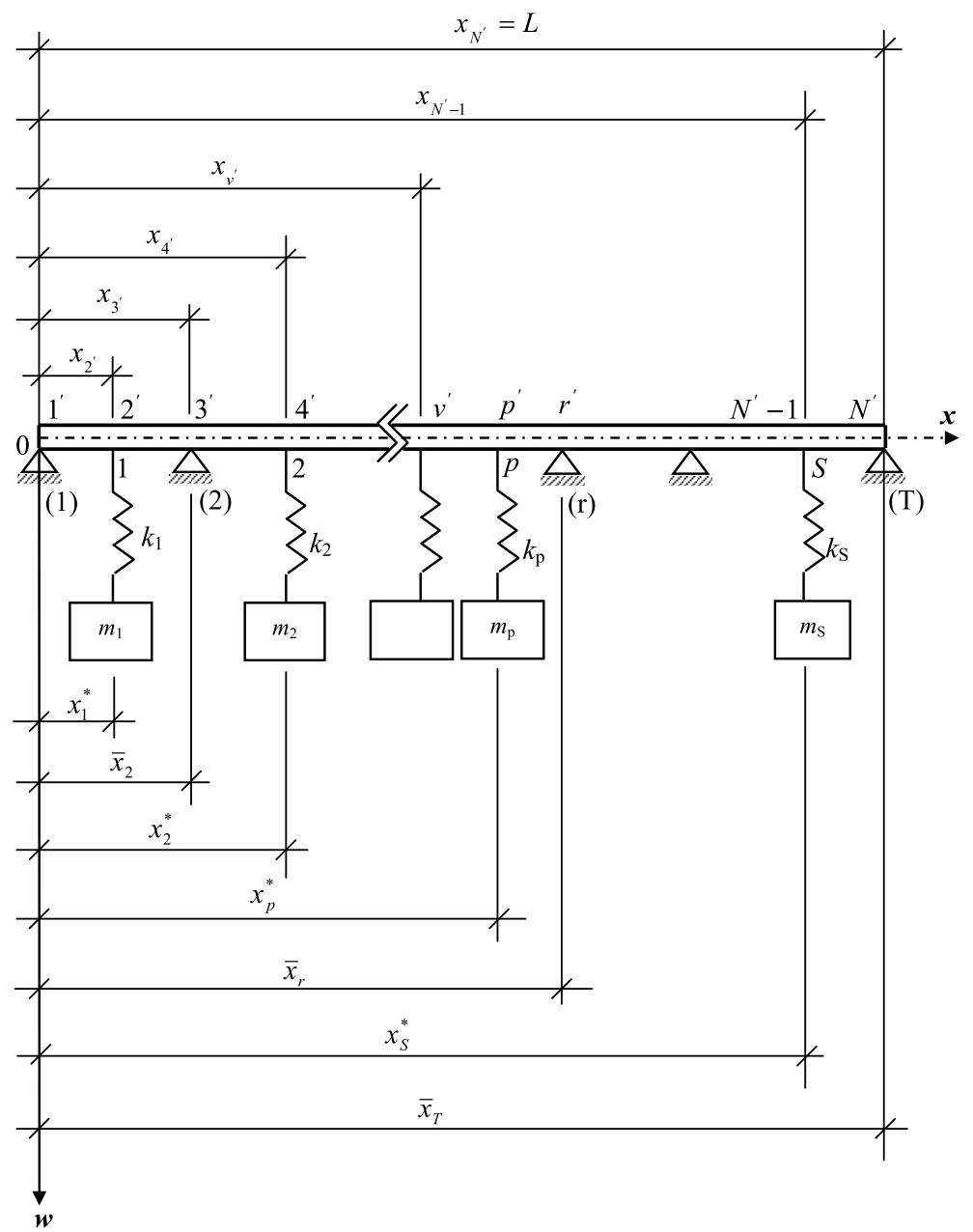

Fig. 2. A Reddy-Bickford uniform beam supported by $T$ pins and carrying $S$ spring-mass systems.

where $\phi(x, t)$ represents the rotation of a normal to the axis of the beam, $m$ is mass per unit length of the beam, $L$ is length of the beam, $A$ is the cross-section area, $I_{x}$ is moment of inertia, $E, G$ are Young's modulus and shear modulus of the beam, respectively, $x$ is the beam position coordinate, $t$ is time variable. The details for the application of Hamilton's principle and the derivation of the equations of motion are presented in Section "Appendix-A" at the end of the paper.

The $p^{\text {th }}$ spring-mass system's equation of motion can be written as:

$$
m_{p} \cdot \ddot{z}_{p}+k_{p} \cdot\left(z_{p}-w_{p}\right)=0
$$

where $m_{p}$ is the mass of the $p^{\text {th }}$ spring-mass system, $k_{p}$ is the spring constant of the $p^{\text {th }}$ spring-mass system, $\ddot{z}_{p}$ and $z_{p}$ are the acceleration and displacement of the $p^{\text {th }}$ spring-mass system relative to its static equilibrium position, respectively, $w_{p}$ is the transverse displacement of the beam at the attaching point of the $p^{\text {th }}$ spring-mass system.

Assuming that the motion is harmonic we substitute for $w(z, t)$ and $\phi(z, t)$ the following:

$$
\begin{aligned}
& w(z, t)=w(z) \cdot \sin (\omega \cdot t) \\
& \phi(z, t)=\phi(z) \cdot \sin (\omega \cdot t) \\
& z_{p}=Z_{p} \cdot \sin (\omega \cdot t)
\end{aligned}
$$

and obtain a system of two coupled ordinary differential equations as: 


$$
\begin{aligned}
& -\frac{68}{105} \cdot \frac{E I_{x}}{L^{2}} \cdot \frac{d^{2} \phi(z)}{d z^{2}}+\frac{16}{105} \cdot \frac{E I_{x}}{L^{3}} \cdot \frac{d^{3} w(z)}{d z^{3}}+\frac{8}{15} \cdot A G \cdot\left[\phi(z)+\frac{1}{L} \cdot \frac{d w(z)}{d z}\right]=0 \\
& m \cdot \omega^{2} \cdot w(z)+\frac{8}{15} \cdot \frac{A G}{L} \cdot\left[\frac{d \phi(z)}{d z}+\frac{1}{L} \cdot \frac{d^{2} w(z)}{d z^{2}}\right]+\frac{16}{105} \cdot \frac{E I_{x}}{L^{3}} \cdot \frac{d^{3} \phi(z)}{d z^{3}}-\frac{1}{21} \cdot \frac{E I_{x}}{L^{4}} \cdot \frac{d^{4} w(z)}{d z^{4}}=0
\end{aligned}
$$

where $z=\frac{x}{L}$ and $\omega$ is natural frequency of the beam systems.

It is assumed that the solutions are:

$$
\begin{aligned}
& w(z)=C \cdot e^{i s z} \\
& \phi(z)=P \cdot e^{i s z}
\end{aligned}
$$

and substituting Eqs (11) and (12) into Eqs (9) and (10) results in

$$
\begin{aligned}
& \left(\frac{8}{15} \cdot A G+\frac{68}{105} \cdot \frac{E I_{x}}{L^{2}} \cdot s^{2}\right) \cdot P+\left(\frac{8}{15} \cdot \frac{A G}{L} \cdot s \cdot i-\frac{16}{105} \cdot \frac{E I_{x}}{L^{3}} \cdot s^{3} \cdot i\right) \cdot C=0 \\
& \left(\frac{8}{15} \cdot \frac{A G}{L} \cdot s \cdot i-\frac{16}{105} \cdot \frac{E I_{x}}{L^{3}} \cdot s^{3} \cdot i\right) \cdot P+\left(m \cdot \omega^{2}-\frac{8}{15} \cdot \frac{A G}{L^{2}} \cdot s^{2}-\frac{1}{21} \cdot \frac{E I_{x}}{L^{4}} \cdot s^{4}\right) \cdot C=0
\end{aligned}
$$

Equations (13) and (14) can be written in matrix form for the two unknowns $P$ and $C$ as

$$
\left[\begin{array}{lc}
\frac{8}{15} \cdot A G+\frac{68}{105} \cdot \frac{E I_{x}}{L^{2}} \cdot s^{2} & \frac{8}{15} \cdot \frac{A G}{L} \cdot s \cdot i-\frac{16}{105} \cdot \frac{E I_{x}}{L^{3}} \cdot s^{3} \cdot i \\
\frac{8}{15} \cdot \frac{A G}{L} \cdot s \cdot i-\frac{16}{105} \cdot \frac{E I_{x}}{L^{3}} \cdot s^{3} \cdot i & m \cdot \omega^{2}-\frac{8}{15} \cdot \frac{A G}{L^{2}} \cdot s^{2}-\frac{1}{21} \cdot \frac{E I_{x}}{L^{4}} \cdot s^{4}
\end{array}\right] \cdot\left\{\begin{array}{l}
P \\
C
\end{array}\right\}=\left\{\begin{array}{l}
0 \\
0
\end{array}\right\}
$$

and a non-trivial solution will be obtained when the determinant of the coefficient matrix will be zero, i.e.

$$
\left[-\frac{4}{525} \cdot \frac{\left(E I_{x}\right)^{2}}{L^{6}}\right] \cdot s^{6}-\left(\frac{8}{15} \cdot \frac{A G \cdot E I_{x}}{L^{4}}\right) \cdot s^{4}+\left(\frac{68}{105} \cdot \frac{E I_{x}}{L^{2}} \cdot m \cdot \omega^{2}\right) \cdot s^{2}+\frac{8}{15} \cdot A G \cdot m \cdot \omega^{2}=0
$$

Thus, there is a sixth-degree polynomial with the unknowns, resulting in six values and the transverse displacement function can be written as:

$$
w(z, t)=\left[C_{1} \cdot e^{i s_{1} z}+C_{2} \cdot e^{i s_{2} z}+C_{3} \cdot e^{i s_{3} z}+C_{4} \cdot e^{i s_{4} z}+C_{5} \cdot e^{i s_{5} z}+C_{6} \cdot e^{i s_{6} z}\right] \cdot \sin (\omega \cdot t)
$$

The expression for bending rotation $w^{\prime}(z, t)$ is given by

$$
w^{\prime}(z, t)=\frac{1}{L} \cdot \frac{d w(z)}{d z} \cdot \sin (\omega \cdot t)
$$

By using Eq. (17), the rotation of normal $\phi(z, t)$ can be obtained as:

$$
\phi(z, t)=-\left(\frac{\gamma \cdot m \cdot \omega^{2}+1}{L}\right) \cdot \frac{d w(z)}{d z} \cdot \sin (\omega \cdot t)-\frac{3}{2} \cdot \frac{\beta}{L^{3}} \cdot \frac{d^{3} w(z)}{d z^{3}} \cdot \sin (\omega \cdot t)+\frac{17}{784} \cdot \frac{\beta^{2}}{L^{5}} \cdot \frac{d^{5} w(z)}{d z^{5}} \cdot \sin (\omega \cdot t)
$$

where

$$
\begin{aligned}
& \beta=\frac{E I_{x}}{A G} \\
& \gamma=\frac{1445}{784} \cdot \frac{\beta}{A G}
\end{aligned}
$$

The shear force function $Q(z, t)$ can be obtained by using Eqs (17) and (19) as:

$$
Q(z, t)=\left[-\frac{8 \cdot A G}{15} \cdot\left(\phi(z)+\frac{1}{L} \cdot \frac{d w(z)}{d z}\right)+\frac{E I_{x}}{21 \cdot L^{3}} \cdot \frac{d^{3} w(z)}{d z^{3}}-\frac{16 \cdot E I_{x}}{105 \cdot L^{2}} \cdot \frac{d^{2} \phi(z)}{d z^{2}}\right] \cdot \sin (\omega \cdot t)
$$

Similarly, the bending moment function $M(z, t)$ can be obtained by using Eqs (17) and (19) as:

$$
M(z, t)=\left(-\frac{E I_{x}}{21 \cdot L^{2}} \cdot \frac{d^{2} w(z)}{d z^{2}}+\frac{16 \cdot E I_{x}}{105 \cdot L} \cdot \frac{d \phi(z)}{d z}\right) \cdot \sin (\omega \cdot t)
$$

The higher-order moment function $M_{h}(z, t)$ can be obtained as:

$$
M_{h}(z, t)=\left(\frac{16 \cdot E I_{x}}{105 \cdot L^{2}} \cdot \frac{d^{2} w(z)}{d z^{2}}-\frac{68 \cdot E I_{x}}{105 \cdot L} \cdot \frac{d \phi(z)}{d z}\right) \cdot \sin (\omega \cdot t)
$$




\section{Determination of natural frequencies and mode shapes}

The state is written due to the values of the transverse displacement $w(z, t)$, bending rotation $w^{\prime}(z, t)$, rotation of normal $\phi(z, t)$, shear force $Q(z, t)$, bending moment $M(z, t)$ and higher-order moment function $M_{h}(z, t)$ at the locations of $z$ and $t$ for Reddy-Bickford beam, as:

$$
\{S(z, t)\}^{T}=\left\langle w(z) w^{\prime}(z) \phi(z) M(z) M_{h}(z) Q(z)\right\rangle \cdot \sin (\omega . t)
$$

where $\{S(z, t)\}$ shows the state vector.

The boundary conditions for the left-end support of the beam are written as:

$$
\begin{aligned}
& w_{1^{\prime}}(z=0)=0 \\
& M_{1^{\prime}}(z=0)=0 \\
& M_{h, 1^{\prime}}(z=0)=0
\end{aligned}
$$

From Eqs (17), (22) and (23), the boundary conditions for the left-end support can be written in matrix equation form as:

$$
\left[B_{1^{\prime}}\right] \cdot\left\{C_{1^{\prime}}\right\}=\{0\}
$$

$$
\left[\begin{array}{cccccc}
1 & 2 & 3 & 4 & 5 & 6 \\
1 & 1 & 1 & 1 & 1 & 1 \\
K_{22} & K_{23} & K_{24} & K_{25} & K_{26} & K_{27} \\
K_{30} & K_{31} & K_{32} & K_{33} & K_{34} & K_{35}
\end{array}\right] \begin{aligned}
& 1 \\
& 2
\end{aligned} \cdot\left\{\begin{array}{l}
C_{1^{\prime}, 1} \\
C_{1^{\prime}, 2} \\
C_{1^{\prime}, 3} \\
C_{1^{\prime}, 4} \\
C_{1^{\prime}, 5} \\
C_{1^{\prime}, 6}
\end{array}\right\}=\left\{\begin{array}{l}
0 \\
0 \\
0
\end{array}\right\}
$$

All coefficients $K_{i}(i=1,2,3, \ldots, 35)$ are presented in Section "Appendix-B" at the end of the paper.

The matching conditions for the $p^{\text {th }}$ intermediate spring-mass system are written by using the continuity of deformations, bending rotations and rotations of normal, and the equilibrium of bending moments, high-order moments and shear forces as (the station numbering corresponding to the $p^{\text {th }}$ intermediate spring-mass system is represented by $\left.p^{\prime}\right)$ :

$$
\begin{aligned}
& w_{p^{\prime}}^{L}\left(z_{p^{\prime}}\right)=w_{p^{\prime}}^{R}\left(z_{p^{\prime}}\right) \\
& w_{p^{\prime}}^{L}\left(z_{p^{\prime}}\right)=w_{p^{\prime}}^{R}\left(z_{p^{\prime}}\right) \\
& \phi_{p^{\prime}}^{L}\left(z_{p^{\prime}}\right)=\phi_{p^{\prime}}^{R}\left(z_{p^{\prime}}\right) \\
& M_{p^{\prime}}^{L}\left(z_{p^{\prime}}\right)=M_{p^{\prime}}^{R}\left(z_{p^{\prime}}\right) \\
& M_{h p^{\prime}}^{L}\left(z_{p^{\prime}}\right)=M_{h p^{\prime}}^{R}\left(z_{p^{\prime}}\right) \\
& Q_{p^{\prime}}^{L}\left(z_{p^{\prime}}\right)-m_{p} \cdot \omega^{2} \cdot Z_{p}=Q_{p^{\prime}}^{R}\left(z_{p^{\prime}}\right)
\end{aligned}
$$

where $L$ and $R$ refer to the left side and right side of the $p^{\text {th }}$ intermediate spring-mass system, respectively.

Substituting Eqs (6) and (8) into Eq. (5) gives:

$$
w_{p^{\prime}}+\left(\alpha_{p}^{2}-1\right) \cdot Z_{p}=0
$$

where $\alpha_{p}=\frac{\omega}{\omega_{p}} ; \omega_{p}=\sqrt{\frac{k_{p}}{m_{p}}}$

From Eqs (17), (18), (19), (21), (22) and (23), the matching conditions for the $p^{\text {th }}$ intermediate spring-mass system can be written in matrix equation form as: 


$$
\left[B_{p^{\prime}}\right] \cdot\left\{C_{p^{\prime}}\right\}=\{0\}
$$

$\left[B_{p^{\prime}}\right]$ coefficient matrix for the $p^{\text {th }}$ intermediate spring-mass system can be written as:

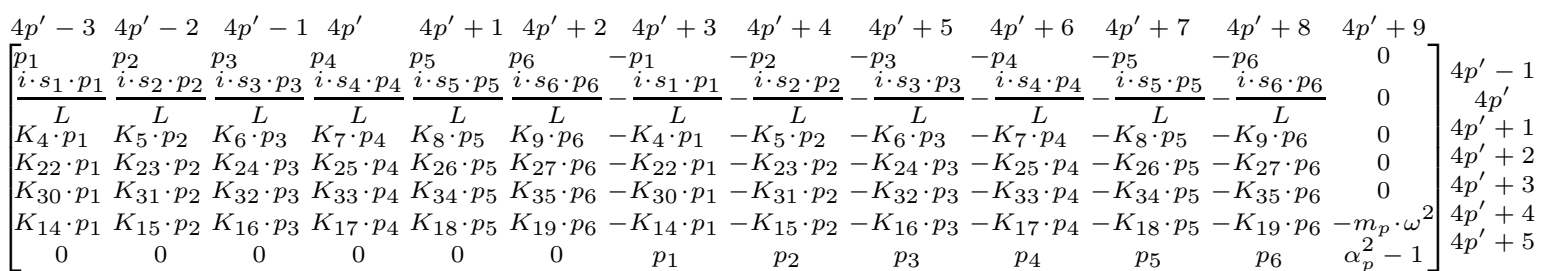

$K_{30} \cdot p_{1} K_{31} \cdot p_{2} K_{32} \cdot p_{3} K_{33} \cdot p_{4} K_{34} \cdot p_{5} K_{35} \cdot p_{6}-K_{30} \cdot p_{1}-K_{31} \cdot p_{2}-K_{32} \cdot p_{3}-K_{33} \cdot p_{4}-K_{34} \cdot p_{5}-K_{35} \cdot p_{6} 004 p^{\prime}+3$

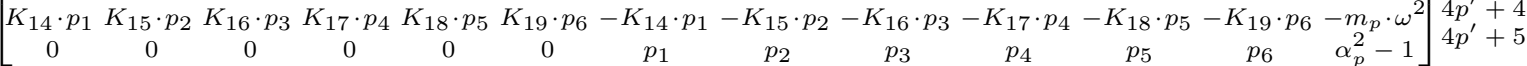

where

$$
p_{1}=e^{\left(i \cdot s_{1} \cdot z_{p^{\prime}}\right)} ; \quad p_{2}=e^{\left(i \cdot s_{2} \cdot z_{p^{\prime}}\right)} ; \quad p_{3}=e^{\left(i \cdot s_{3} \cdot z_{p^{\prime}}\right)} ; \quad p_{4}=e^{\left(i \cdot s_{4} \cdot z_{p^{\prime}}\right)} ; \quad p_{5}=e^{\left(i \cdot s_{5} \cdot z_{p^{\prime}}\right)} ; \quad p_{6}=e^{\left(i \cdot s_{6} \cdot z_{p^{\prime}}\right)}
$$

The matching conditions for the $r^{\text {th }}$ support are written by using continuity of deformations, bending rotations and rotations of normal, and the equilibrium of bending moments, high-order moments and shear forces, as (the station numbering corresponding to the $r^{\text {th }}$ intermediate support is represented by $r^{\prime}$ ):

$$
\begin{aligned}
& w_{r^{\prime}}^{L}\left(z_{r^{\prime}}\right)=w_{r^{\prime}}^{R}\left(z_{r^{\prime}}\right)=0 \\
& w_{r^{\prime}}^{L}\left(z_{r^{\prime}}\right)=w_{r^{\prime}}^{R}\left(z_{r^{\prime}}\right) \\
& \phi_{r^{\prime}}^{L}\left(z_{r^{\prime}}\right)=\phi_{r^{\prime}}^{R}\left(z_{r^{\prime}}\right) \\
& M_{r^{\prime}}^{L}\left(z_{r^{\prime}}\right)=M_{r^{\prime}}^{R}\left(z_{r^{\prime}}\right) \\
& M_{h r^{\prime}}^{L}\left(z_{r^{\prime}}\right)=M_{h r^{\prime}}^{R}\left(z_{r^{\prime}}\right)
\end{aligned}
$$

From Eqs (17), (18), (19), (22) and (23), the matching conditions for the $r^{\text {th }}$ intermediate support can be written in matrix equation form as:

$$
\left[B_{r^{\prime}}\right] \cdot\left\{C_{r^{\prime}}\right\}=\{0\}
$$

$\left[B_{r^{\prime}}\right]$ coefficient matrix for the $p^{\text {th }}$ intermediate spring-mass system can be written as:

$$
\begin{gathered}
\left.\begin{array}{cccccccccccc}
4 r^{\prime}-3 & 4 r^{\prime}-2 & 4 r^{\prime}-1 & 4 r^{\prime} & 4 r^{\prime}+1 & 4 r^{\prime}+2 & 4 r^{\prime}+3 & 4 r^{\prime}+4 & 4 r^{\prime}+5 & 4 r^{\prime}+6 & 4 r^{\prime}+7 & 4 r^{\prime}+8 \\
r_{1} & r_{2} & r_{3} & r_{4} & r_{5} & r_{6} & 0 & 0 & 0 & 0 & 0 & 0 \\
0 & 0 & 0 & 0 & 0 & 0 & r_{1} & r_{2} & r_{3} & r_{4} & r_{5} & r_{6} \\
i \cdot s_{1} \cdot r_{1} \\
\hline L & \frac{i \cdot s_{2} \cdot r_{2}}{L} & \frac{i \cdot s_{3} \cdot r_{3}}{L} & \frac{i \cdot s_{4} \cdot r_{4}}{L} & \frac{i \cdot s_{5} \cdot r_{5}}{L} & \frac{i \cdot s_{6} \cdot r_{6}}{L} & -\frac{i \cdot s_{1} \cdot r_{1}}{L} & -\frac{i \cdot s_{2} \cdot r_{2}}{L} & -\frac{i \cdot s_{3} \cdot r_{3}}{L} & -\frac{i \cdot s_{4} \cdot r_{4}}{L} & -\frac{i \cdot s_{5} \cdot r_{5}}{L}-\frac{i \cdot s_{6} \cdot r_{6}}{L} & 4 r^{\prime} \\
K_{4} \cdot r_{1} & K_{5} \cdot r_{2} & K_{6} \cdot r_{3} & K_{7} \cdot r_{4} & K_{8} \cdot r_{5} & K_{9} \cdot r_{6} & -K_{4} \cdot r_{1} & -K_{5} \cdot r_{2} & -K_{6} \cdot r_{3} & -K_{7} \cdot r_{4} & -K_{8} \cdot r_{5} & -K_{9} \cdot r_{6} \\
K_{22} \cdot r_{1} & K_{23} \cdot r_{2} & K_{24} \cdot r_{3} & K_{25} \cdot r_{4} & K_{26} \cdot r_{5} & K_{27} \cdot r_{6} & -K_{22} \cdot r_{1} & -K_{23} \cdot r_{2} & -K_{24} \cdot r_{3} & -K_{25} \cdot r_{4} & -K_{26} \cdot r_{5} & -K_{27} \cdot r_{6} \\
K_{30} \cdot r_{1} & K_{31} \cdot r_{2} & K_{32} \cdot r_{3} & K_{33} \cdot r_{4} & K_{34} \cdot r_{5} & K_{35} \cdot r_{6} & -K_{30} \cdot r_{1} & -K_{31} \cdot r_{2} & -K_{32} \cdot r_{3} & -K_{33} \cdot r_{4} & -K_{34} \cdot r_{5} & -K_{35} \cdot r_{6}
\end{array}\right] 4 r^{\prime}+4
\end{gathered}
$$

where

$$
r_{1}=e^{\left(i \cdot s_{1} \cdot z_{r^{\prime}}\right)} ; \quad r_{2}=e^{\left(i \cdot s_{2} \cdot z_{r^{\prime}}\right)} ; \quad r_{3}=e^{\left(i \cdot s_{3} \cdot z_{r^{\prime}}\right)} ; \quad r_{4}=e^{\left(i \cdot s_{4} \cdot z_{r^{\prime}}\right)} ; \quad r_{5}=e^{\left(i \cdot s_{5} \cdot z_{r^{\prime}}\right)} ; \quad r_{6}=e^{\left(i \cdot s_{6} \cdot z_{r^{\prime}}\right)}
$$

The boundary conditions for the right-end support of the beam are written as:

$$
\begin{aligned}
& w_{N^{\prime}}(z=1)=0 \\
& M_{N^{\prime}}(z=1)=0 \\
& M_{h, N^{\prime}}(z=1)=0
\end{aligned}
$$

From Eqs (17), (22) and (23), the boundary conditions for the right-end support can be written in matrix equation form as:

$$
\left[B_{N^{\prime}}\right] \cdot\left\{C_{N^{\prime}}\right\}=\{0\}
$$




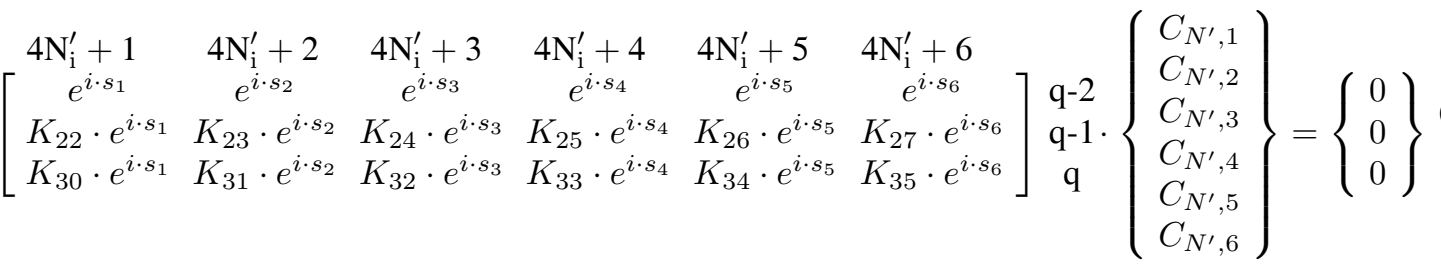

where $N_{i}^{\prime}$ is the total number of intermediate stations and is given by:

$$
N_{i}^{\prime}=N^{\prime}-2
$$

with

$$
N^{\prime}=S+T
$$

In Eq. (37), $N^{\prime}$ is the total number of stations.

The total number of equations for the integration constants is obtained as:

$$
q=3+6 \cdot(T-2)+7 \cdot S+3
$$

From Eq. (38), it can be seen that; the left-end support of the beam has three equations, each intermediate support of the beam has six equations, each intermediate spring-mass system of the beam has seven equations and the right-end support of the beam has three equations.

In this paper, by using Eqs (26b), (30), (33) and (35b), the coefficient matrices for left-end support, each intermediate spring-mass system, each intermediate pinned support and right-end support of a Reddy-Bickford beam are derived, respectively. In the next step, the numerical assembly technique is used to establish the overall coefficient matrix for the whole vibrating system as given in Eq. (39). In the last step, for non-trivial solution, equating the last overall coefficient matrix to zero one determines the natural frequencies of the vibrating system as given in Eq. (40); and substituting of the last integration constants into the related eigenfunctions one determines the associated mode shapes.

$$
\begin{aligned}
& {[B] \cdot\{C\}=\{0\}} \\
& |B|=0
\end{aligned}
$$

\section{Numerical analysis and discussions}

Natural frequencies are found by determining values for which the determinant of the coefficient matrix is equal to zero. There are various methods for calculating the roots of the frequency equation. One common used and simple technique is the secant method in which a linear interpolation is employed.

For numerical analysis, four examples are considered. For four examples, natural frequencies of the beam system, $\omega_{i}(i=1,2,3,4,5)$ are calculated by using a computer program prepared by author. In this program, the secant method is used in which determinant values are evaluated for a range $\left(\omega_{i}\right)$ values. The $\left(\omega_{i}\right)$ value causing a sign change between the successive determinant values is a root of frequency equation and means a frequency for the system.

The four numerical results of this paper are obtained based on a uniform, rectangular Reddy-Bickford and Timoshenko beams with the following data as:

$h=0.30 \mathrm{~m} ; b=0.25 \mathrm{~m} ; E=2.1 \times 10^{8} \mathrm{kN} / \mathrm{m}^{2} ; G=8.1 \times 10^{7} \mathrm{kN} / \mathrm{m}^{2} ; m=0.30 \mathrm{kN} . \mathrm{sec}^{2} / \mathrm{m} ; L=3.0 \mathrm{~m} ;$ total mass $m_{b}=m \cdot L=0.90 \mathrm{kN} \cdot \sec ^{2}$; reference spring constant $k_{b}=E I_{x} / L^{3}=4375 \mathrm{kN} / \mathrm{m}$.

In literature, many values for the shear correction factor $k$ were suggested, but in this paper, the original values suggested by Timoshenko $k=\frac{5}{6}$ and $k=\frac{14}{17}$ [5] are used for Timoshenko beams.

All numerical results are given for the following three models: Timoshenko model with two values for shear correction factor $k$ and Reddy-Bickford model. 
Table 1

Natural frequencies of the three spring-mass systems with respect to the static beam

\begin{tabular}{lccc}
\hline Numbering, $p$ & 1 & 2 & 3 \\
\hline$\hat{m}_{p}=m_{p} / m_{b}$ & 0.2 & 0.5 & 1.0 \\
$\hat{k}_{p}=k_{p} / k_{b}$ & 3.0 & 4.5 & 6.0 \\
$\omega_{p}=\sqrt{k_{p} / m_{p}}$ & 467.7072 & 362.2844 & 295.8040 \\
\hline
\end{tabular}

Table 2

The first five natural frequencies of the uniform single-span pinned-pinned Reddy-Bickford and Timoshenko beams

\begin{tabular}{clrrrrr}
\hline Cases & Methods & \multicolumn{5}{c}{ Natural frequencies, $\omega_{i}(\mathrm{rad} / \mathrm{sec})$} \\
\cline { 3 - 6 } & & $\omega_{1}$ & \multicolumn{1}{c}{$\omega_{2}$} & \multicolumn{1}{c}{$\omega_{3}$} & \multicolumn{1}{c}{$\omega_{4}$} & $\omega_{5}$ \\
\hline 1 & RBT & 484.8633 & 657.1381 & 2616.0103 & 5583.6113 & 9280.5909 \\
& TBT $(k=5 / 6)$ & 453.8104 & 698.4874 & 2627.3528 & 5584.8120 & 9274.0509 \\
& TBT $(k=14 / 17)$ & 453.8024 & 698.3930 & 2625.9085 & 5578.6028 & 9258.0570 \\
2 & RBT & 304.2607 & 392.8831 & 474.4894 & 605.4208 & 2606.5611 \\
& TBT $(k=5 / 6)$ & 285.5570 & 345.1291 & 465.3434 & 736.5435 & 2636.6463 \\
& TBT $(k=14 / 17)$ & 285.5515 & 345.1226 & 465.3412 & 736.4541 & 2635.2070 \\
\hline
\end{tabular}

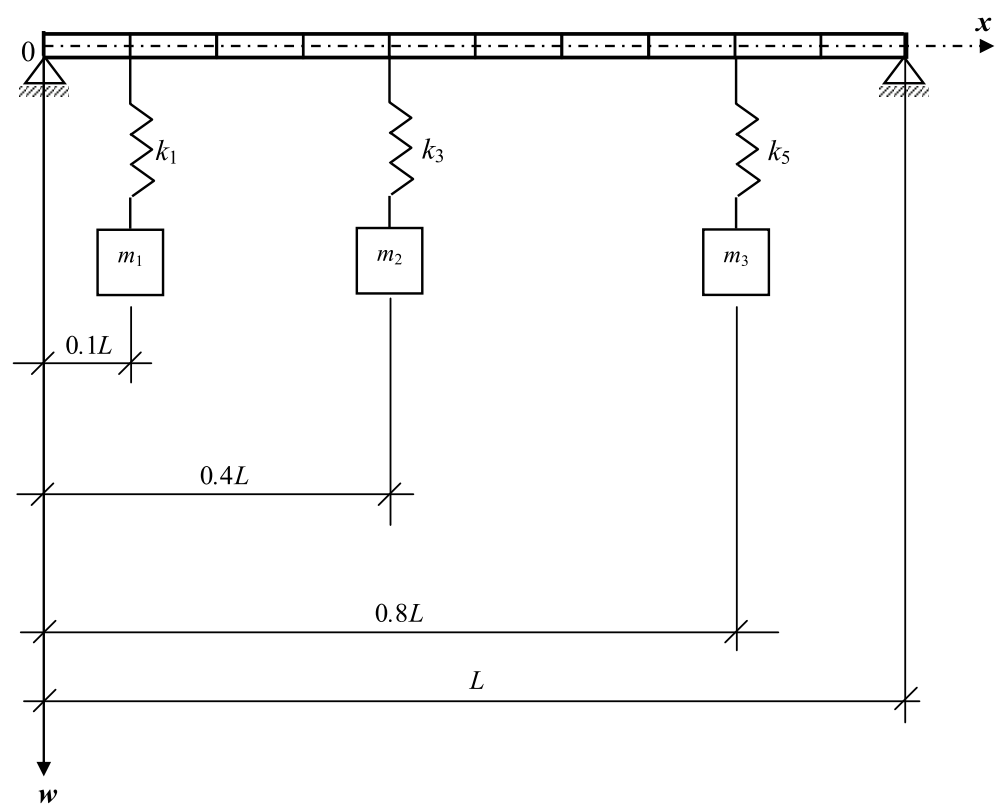

Fig. 3. A single-span pinned-pinned Reddy-Bickford beam carrying three intermediate spring-mass systems, $p=1$ to 3 .

\subsection{Free vibration analysis of the uniform single-span pinned-pinned Reddy-Bickford beam carrying three intermediate spring-mass systems}

In the first numerical example, the uniform single-span pinned-pinned Reddy-Bickford beam carrying one and three intermediate spring-mass systems, respectively, are considered. For this numerical example, the natural frequencies of the static beam without any spring-mass systems (see Fig. 3) are given in (Table 1). The frequency values obtained for the first five modes are presented in (Table 2) by comparing with the frequency values obtained for Timoshenko beam, for two cases, which are presented below:

1st case: In this case, there is one spring-mass system and $z_{p}^{*}=x_{p}^{*} / L=0.75, p=1$;

2nd case: In this case, there are three spring-mass systems and $z_{p}^{*}=0.1,0.4$ and 0.8 , respectively, $p=1,2,3$. 


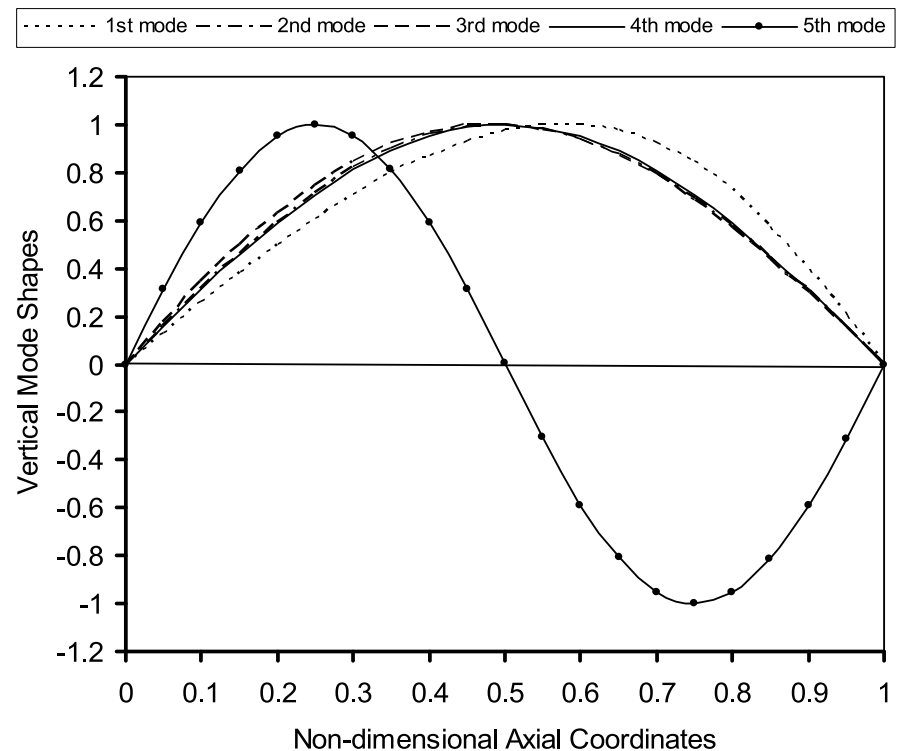

Fig. 4. The first five mode shapes for the second case.

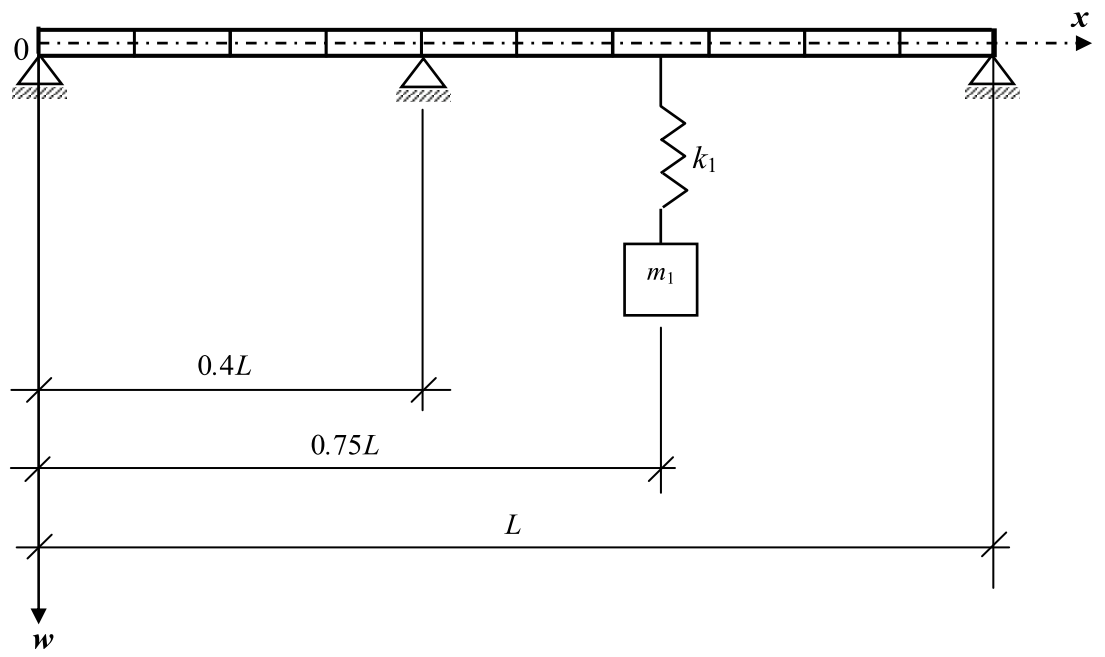

Fig. 5. A two-span Reddy-Bickford beam carrying one intermediate spring-mass system.

For the second case, the first five mode shapes are shown in (Fig. 4).

Figure 4 indicates that the first four mode shapes are similar since the fourth mode frequency of the beam with three attachments is very close to the first mode frequency of the beam with no attachments.

\subsection{Free vibration analysis of the uniform two-span Reddy-Bickford beam carrying one intermediate spring-mass} system

In the second numerical example (see Fig. 5), the uniform two-span Reddy-Bickford beam carrying one springmass system is considered. In this numerical example, for the intermediate support, $\bar{z}_{1}=0.4$; for the intermediate spring-mass system, $z_{1}^{*}=0.75, \hat{m}_{p}=m_{p} / m_{b}=0.2$ and $\hat{k}_{p}=k_{p} / k_{b}=3.0$. The frequency values obtained for the first five modes are presented in (Table 3 ) by comparing with the frequency values obtained for Timoshenko beam and mode shapes for the model with one spring-mass system of Reddy-Bickford beam are presented in (Fig. 6). 
Table 3

The first five natural frequencies of the uniform two-span Reddy-Bickford and Timoshenko beams

\begin{tabular}{clrrrrr}
\hline \multirow{2}{*}{$\begin{array}{c}\text { No. of spring- } \\
\text { mass system, } p\end{array}$} & Methods & \multicolumn{5}{c}{ Natural frequencies, $\omega_{i}(\mathrm{rad} / \mathrm{sec})$} \\
\cline { 3 - 7 } & & $\omega_{1}$ & $\omega_{2}$ & $\omega_{3}$ & \multicolumn{1}{c}{$\omega_{4}$} & $\omega_{5}$ \\
\hline 1 & RBT & 470.2050 & 2197.8545 & 4764.4068 & 7456.4055 & 13453.9399 \\
& TBT $(k=5 / 6)$ & 465.2437 & 2218.4578 & 4755.2479 & 7429.0908 & 13453.1755 \\
& TBT $(k=14 / 17)$ & 465.2400 & 2217.1056 & 4749.1944 & 7415.7855 & 13404.0870 \\
0 & RBT & 2208.8649 & 4765.0083 & 7456.7652 & 13454.4826 & 14808.5122 \\
& TBT $(k=5 / 6)$ & 2207.5220 & 4754.6557 & 7428.7194 & 13434.6323 & 14645.2992 \\
& TBT $(k=14 / 17)$ & 2206.1621 & 4748.6033 & 7415.4122 & 13403.5425 & 14601.6668 \\
\hline
\end{tabular}

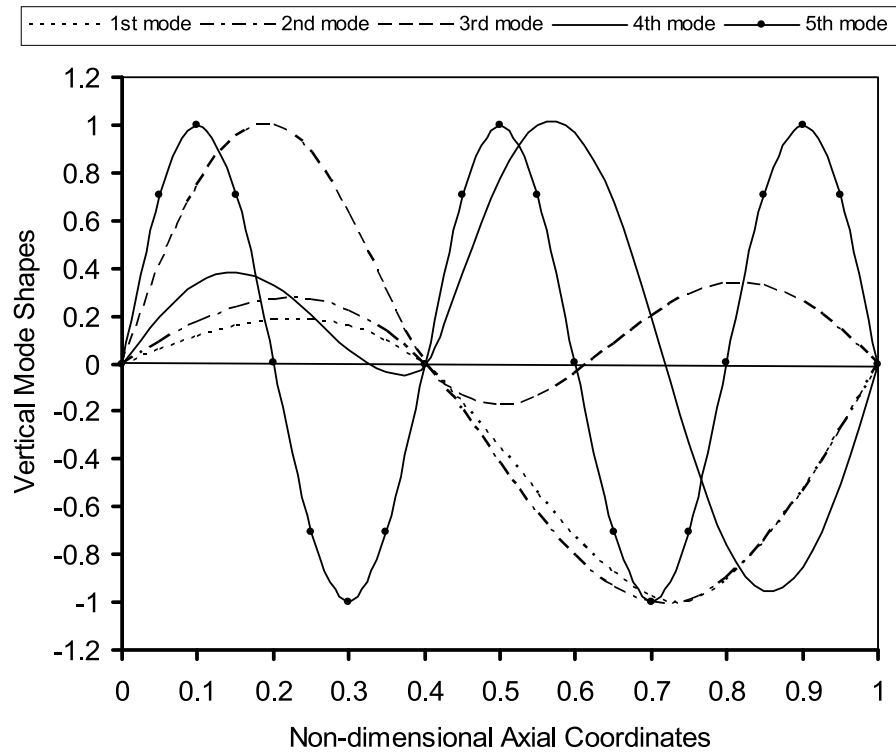

Fig. 6. The first five mode shapes for the model with one spring-mass system of Reddy-Bickford beam.

The first two mode shapes have similar forms in (Fig. 5); this is because the frequency values for the 2nd, 3rd, 4th and 5th modes of the beam with one attachment are very close to the ones for the 1st, 2nd, 3rd and 4th modes of the beam with no attachments.

\subsection{Free vibration analysis of the uniform three-span Reddy-Bickford beam carrying three intermediate spring-mass systems}

In the third numerical example (see Fig. 7), the uniform three-span Reddy-Bickford beam carrying three springmass systems is considered. In this numerical example, for the first intermediate support, $\bar{z}_{1}=0.3$; for the second intermediate support, $\bar{z}_{2}=0.7$; for the first intermediate spring-mass system, $z_{1}^{*}=0.1, \hat{m}_{1}=0.2$ and $\hat{k}_{1}=3.0$; for the second intermediate spring-mass system, $z_{2}^{*}=0.4, \hat{m}_{2}=0.3$ and $\hat{k}_{2}=3.5$; for the third intermediate spring-mass system, $z_{3}^{*}=0.8, \hat{m}_{3}=0.5$ and $\hat{k}_{3}=4.5$. The frequency values obtained for the first five modes are presented in (Table 4) by comparing with the frequency values obtained for Timoshenko beam and mode shapes for the model with three spring-mass systems of Reddy-Bickford beam are presented in (Fig. 8).

From (Table 4) one sees that the 4th and 5th mode frequency values are very close to the 1 st and 2 nd modes values obtained for the model with no attachment.

\subsection{Free vibration analysis of the uniform four-span Reddy-Bickford beam carrying three intermediate spring-mass systems}

In the fourth numerical example (see Fig. 9), the uniform four-span Reddy-Bickford beam carrying three springmass systems is considered. In this numerical example, for the first intermediate support, $\bar{z}_{1}=0.3$; for the second 
Table 4

The first five natural frequencies of the uniform three-span Reddy-Bickford and Timoshenko beams

\begin{tabular}{clrrrrr}
\hline No. of spring- & Methods & \multicolumn{5}{c}{ Natural frequencies, $\omega_{i}(\mathrm{rad} / \mathrm{sec})$} \\
\cline { 3 - 6 } mass system, $p$ & & \multicolumn{1}{c}{$\omega_{1}$} & \multicolumn{1}{c}{$\omega_{2}$} & \multicolumn{1}{c}{$\omega_{3}$} & \multicolumn{1}{c}{$\omega_{4}$} & $\omega_{5}$ \\
\hline 3 & RBT & 362.6716 & 412.8784 & 468.0770 & 5065.4472 & 7738.3184 \\
& TBT $(k=5 / 6)$ & 361.8885 & 412.0710 & 467.3327 & 5065.7704 & 7717.3108 \\
& TBT $(k=14 / 17)$ & 361.8867 & 412.0693 & 467.3312 & 5059.3155 & 7702.8465 \\
0 & RBT & 5070.4999 & 7744.1261 & 8525.4795 & 15026.1236 & 21683.8047 \\
& TBT $(k=5 / 6)$ & 5060.7449 & 7711.4823 & 8440.2629 & 14841.1120 & 21492.0606 \\
& TBT $(k=14 / 17)$ & 5054.2869 & 7697.0066 & 8419.1167 & 14795.5666 & 21419.3446 \\
\hline
\end{tabular}

Table 5

The first five natural frequencies of the uniform four-span Reddy-Bickford and Timoshenko beams

\begin{tabular}{clrrrrr}
\hline No. of spring- & Methods & \multicolumn{5}{c}{ Natural frequencies, $\omega_{i}(\mathrm{rad} / \mathrm{sec})$} \\
\cline { 3 - 6 } mass system, $p$ & & \multicolumn{1}{c}{$\omega_{1}$} & \multicolumn{1}{c}{$\omega_{2}$} & $\omega_{3}$ & \multicolumn{1}{c}{$\omega_{4}$} & $\omega_{5}$ \\
\hline 3 & RBT & 362.6420 & 412.6374 & 468.0605 & 7738.3157 & 7825.7439 \\
& TBT $(k=5 / 6)$ & 361.9167 & 412.3122 & 467.3481 & 7717.3066 & 7787.9414 \\
& TBT $(k=14 / 17)$ & 361.9148 & 412.3111 & 467.3466 & 7702.8422 & 7719.3680 \\
0 & RBT & 7744.1261 & 7771.3064 & 15026.1236 & 16116.2922 & 22048.3851 \\
& TBT $(k=5 / 6)$ & 7711.4823 & 7782.1615 & 14841.1120 & 17405.7245 & 21716.2060 \\
& TBT $(k=14 / 17)$ & 7697.0066 & 7766.1442 & 14795.5666 & 17336.4168 & 21638.4929 \\
\hline
\end{tabular}

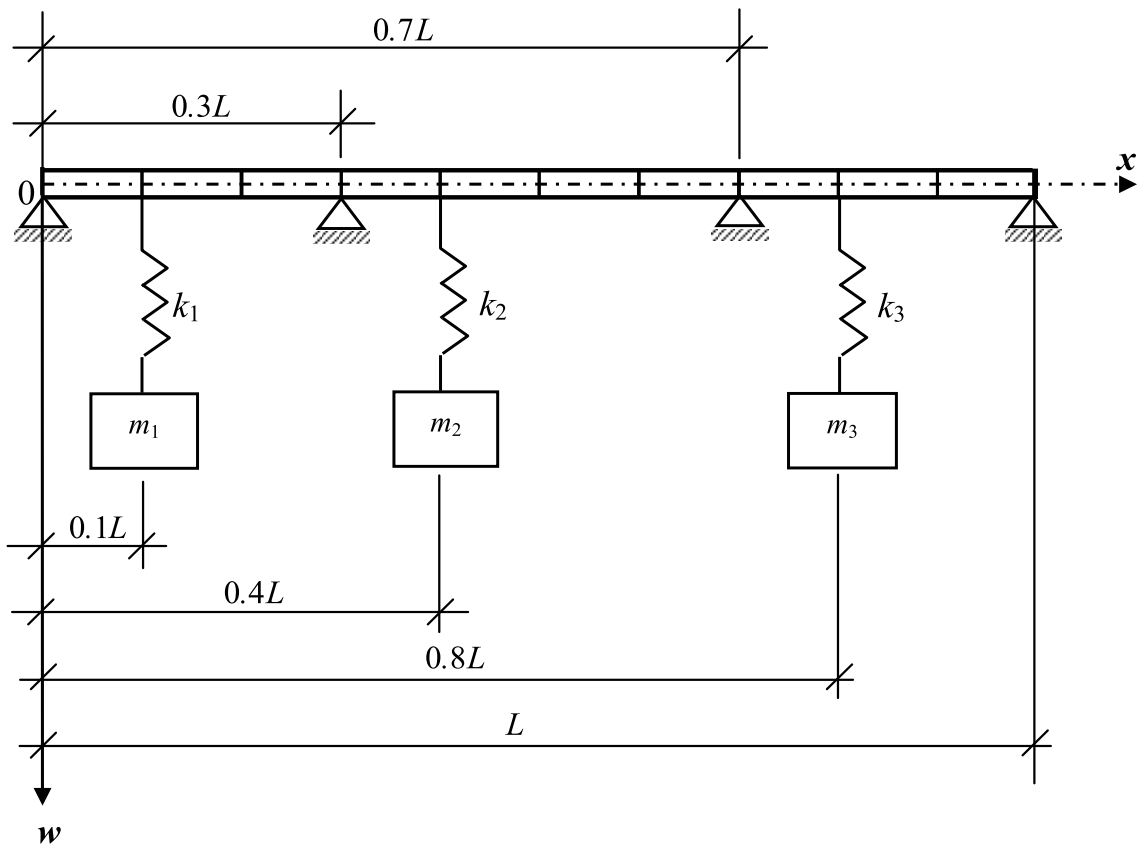

Fig. 7. A three-span Reddy-Bickford beam carrying three intermediate spring-mass systems.

intermediate support, $\bar{z}_{2}=0.5$; for the third intermediate support $\bar{z}_{3}=0.7$; for the first, the second and the third intermediate spring-mass systems, locations and non-dimensional parameters are taken as which are given in Section 3.3. The frequency values obtained for the first five modes are presented in (Table 5) by comparing with the frequency values obtained for Timoshenko beam and mode shapes for the model with three spring-mass systems of four-span Reddy-Bickford beam are shown in (Fig. 10).

It is seen from (Table 5) that, as in Section 3.3, the 4th and 5th mode frequency values are very close to the 1 st and 2 nd modes frequency values obtained for the model with no attachment. 


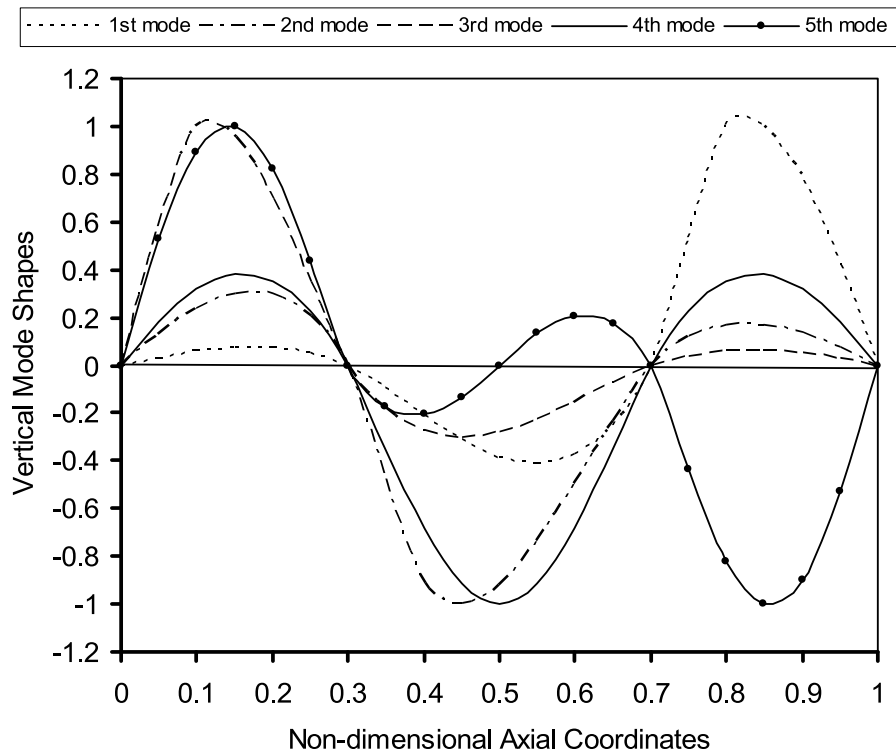

Fig. 8. The first five mode shapes for the model with three spring-mass systems of Reddy-Bickford beam.

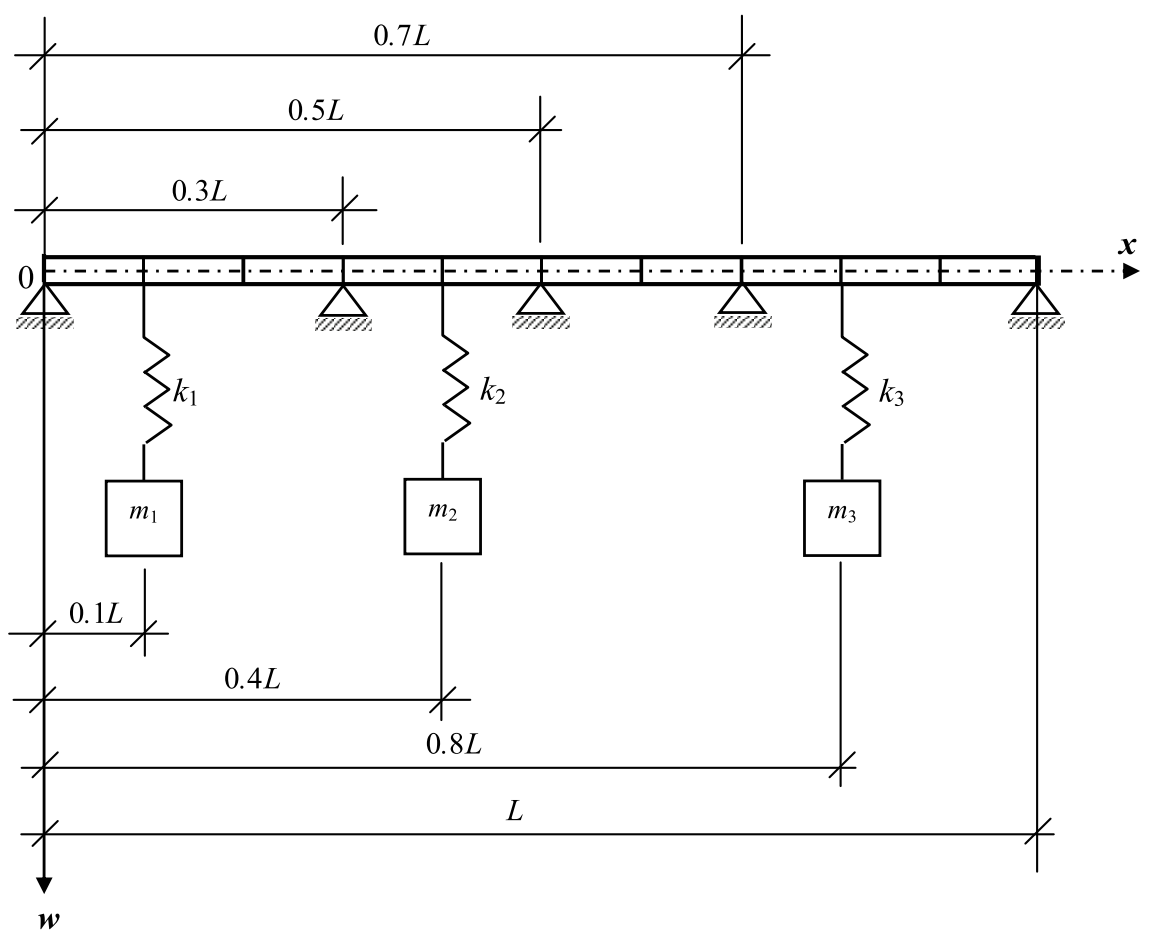

Fig. 9. A four-span Reddy-Bickford beam carrying three intermediate spring-mass systems.

\section{Conclusion}

In this study, the frequency values and mode shapes for the free vibration of the multi-span Reddy-Bickford beam with multiple spring-mass systems are obtained for different number of spans and spring-masses with different locations. In four numerical examples, the frequency values are presented in tables with the values obtained for 


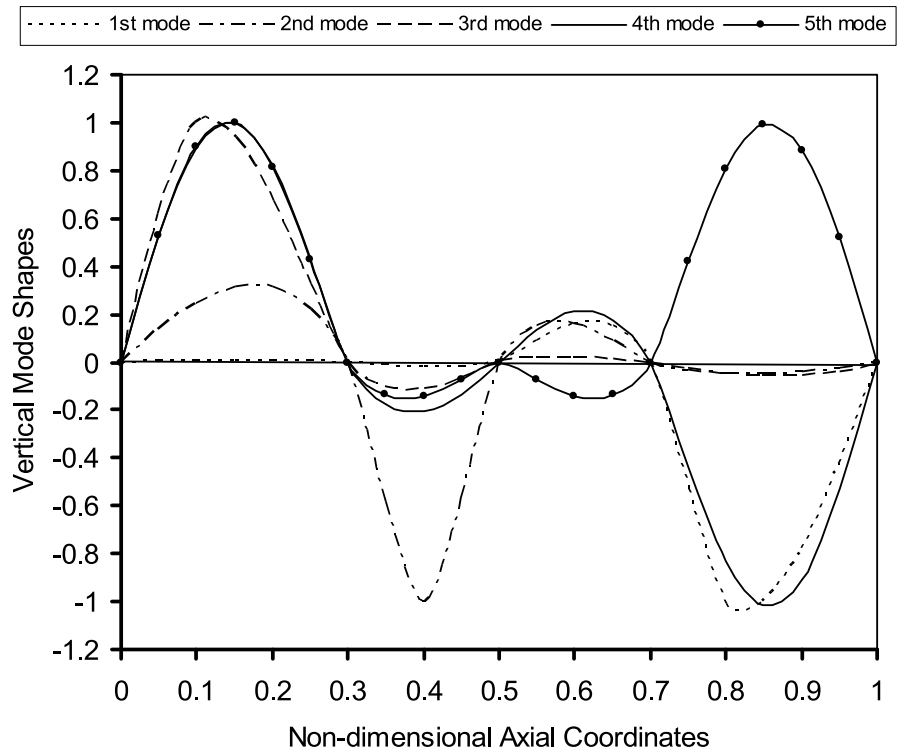

Fig. 10. The first five mode shapes for the model with three spring-mass systems of four-span Reddy-Bickford beam.

Reddy-Bickford and Timoshenko beams.

For all numerical examples, the differences between natural frequency values of Timoshenko beam models with $k=\frac{5}{6}$ and $k=\frac{14}{17}$ are small.

Generally, the frequency values of the multi-span Reddy-Bickford beams carrying no spring-mass system are higher than the values of the multi-span Timoshenko beams carrying no spring-mass system. But, as the number of spring-mass systems $(p)$ is increased and the frequency values of the multi-span Reddy-Bickford beams carrying spring-mass system are compared with the frequency values of the multi-span Timoshenko beams carrying springmass system, very good agreement is observed. It can be seen from the tables that, the differences between ReddyBickford beams and Timoshenko beams become more pronounced for the higher frequencies. So that, to be on safe side it is recommended to use the higher-order theory.

It can be seen from the tables that, the frequency values show a very high decrease as a spring-mass system is attached to the bare beam; this decrease shows a continuity as the number of spring-mass attachments is increased, for Reddy-Bickford and Timoshenko beam theories.

It is, also, seen from the tables and the mode shapes that one can use the relation $\omega_{p+i} \approx \omega_{b i}(i=1,2,3, \ldots)$ for Reddy-Bickford and Timoshenko beams with spring-mass attachments where $p$ is the number of spring-mass attachments, $i$ is the number of modes considered and $\omega_{b}$ is the frequency of the beam carrying no spring-mass system. Therefore, the first $(p+i)$ mode shapes of the beam carrying $p$ spring-mass attachments have similar forms since the $(p+i)^{\text {th }}$ mode frequency value of the beam with attachments is very close to the 1 st mode frequency value of the beam with no attachment.

\section{Appendix A}

The details for the application of Hamilton's principle and the derivation of the equations of motion are presented below.

The displacements according to Reddy-Bickford beam theory can be written as:

$$
\begin{aligned}
& u(x, y, t)=y \cdot \phi(x, t)-\alpha \cdot y^{3} \cdot\left[\phi(x, t)+\frac{\partial w(x, t)}{\partial x}\right] \\
& w(x, y, t)=w_{0}(x, t)
\end{aligned}
$$


The strain-displacement relations of Reddy-Bickford beam theory can be written as:

$$
\begin{aligned}
\varepsilon_{x x} & =\frac{\partial u}{\partial x} \\
\gamma_{x y} & =\frac{\partial u}{\partial y}+\frac{\partial w}{\partial x}
\end{aligned}
$$

Equations (A.3) and (A.4) can be rewritten by using Eqs. (A.1) and (A.2) as:

$$
\begin{aligned}
& \varepsilon_{x x}=y \cdot \frac{\partial \phi(x, t)}{\partial x}-\alpha \cdot y^{3} \cdot\left(\frac{\partial \phi(x, t)}{\partial x}+\frac{\partial^{2} w(x, t)}{\partial x^{2}}\right) \\
& \gamma_{x y}=\phi(x, t)-\beta \cdot y^{2} \cdot\left(\phi(x, t)+\frac{\partial w(x, t)}{\partial x}\right)+\frac{\partial w(x, t)}{\partial x}
\end{aligned}
$$

where

$$
\beta=3 \cdot \alpha=\frac{4}{h^{2}}
$$

The virtual kinetic energy $\delta V$ and the virtual potential energy $\delta \Pi$ can be written as:

$$
\begin{aligned}
& \delta V=\int_{0}^{L} m \cdot \frac{\partial w(x, t)}{\partial t} \cdot \frac{\partial \delta w(x, t)}{\partial t} d x \\
& \delta \prod=\int_{0}^{L} \int_{A}\left(\sigma_{x x} \cdot \delta \varepsilon_{x x}+\sigma_{x y} \cdot \delta \gamma_{x y}\right) \cdot d A \cdot d x
\end{aligned}
$$

where $\sigma_{x x}$ is the normal stress and $\sigma_{x y}$ is the transverse shear stress. These stresses can be obtained as:

$$
\begin{aligned}
& \sigma_{x x}=E \cdot \varepsilon_{x x} \\
& \sigma_{x y}=G \cdot \gamma_{x y}
\end{aligned}
$$

The equations of motion for Reddy-Bickford beam are derived by applying Hamilton's principle, which is given by

$$
\delta \int_{t_{1}}^{t_{2}} \int_{0}^{L} L_{g} \cdot d x \cdot d t=0
$$

where

$$
L_{g}=V-\prod
$$

is termed as the Lagrangian density function.

By taking the variation of the Lagrangian density function; integrating Eq. (A.12) by parts, and then collecting all the terms of the integrand with respect to $\delta w(x, t)$ and $\delta \phi(x, t)$, one can derive the following equations of motion as the coefficients of $\delta w(x, t)$ and $\delta \phi(x, t)$ :

$$
\begin{aligned}
& -\frac{68}{105} \cdot E I_{x} \cdot \frac{\partial^{2} \phi(x, t)}{\partial x^{2}}+\frac{16}{105} \cdot E I_{x} \cdot \frac{\partial^{3} w(x, t)}{\partial x^{3}}+\frac{8}{15} \cdot A G \cdot\left[\phi(x, t)+\frac{\partial w(x, t)}{\partial x}\right]=0 \\
& -m \cdot \frac{\partial^{2} w(x, t)}{\partial t^{2}}+\frac{8}{15} \cdot A G \cdot\left[\frac{\partial \phi(x, t)}{\partial x}+\frac{\partial^{2} w(x, t)}{\partial x^{2}}\right]+\frac{16}{105} \cdot E I_{x} \cdot \frac{\partial^{3} \phi(x, t)}{\partial x^{3}}-\frac{1}{21} \cdot E I_{x} \cdot \frac{\partial^{4} w(x, t)}{\partial x^{4}}=0(\mathrm{~A}
\end{aligned}
$$




\section{Appendix B}

The following coefficients $K_{i}(i=1,2,3, \ldots, 35)$ are used in this paper.

$$
\begin{aligned}
& K_{1}=-\frac{\gamma \cdot m \cdot \omega^{2}+1}{L} \\
& K_{2}=-\frac{3}{2} \cdot \frac{\beta}{L^{3}} \\
& K_{3}=\frac{17}{784} \cdot \frac{\beta^{2}}{L^{5}} \\
& K_{4}=K_{1} \cdot i \cdot s_{1}-K_{2} \cdot i \cdot s_{1}^{3}+K_{3} \cdot i \cdot s_{1}^{5} \\
& K_{5}=K_{1} \cdot i \cdot s_{2}-K_{2} \cdot i \cdot s_{2}^{3}+K_{3} \cdot i \cdot s_{2}^{5} \\
& K_{6}=K_{1} \cdot i \cdot s_{3}-K_{2} \cdot i \cdot s_{3}^{3}+K_{3} \cdot i \cdot s_{3}^{5} \\
& K_{7}=K_{1} \cdot i \cdot s_{4}-K_{2} \cdot i \cdot s_{4}^{3}+K_{3} \cdot i \cdot s_{4}^{5} \\
& K_{8}=K_{1} \cdot i \cdot s_{5}-K_{2} \cdot i \cdot s_{5}^{3}+K_{3} \cdot i \cdot s_{5}^{5} \\
& K_{9}=K_{1} \cdot i \cdot s_{6}-K_{2} \cdot i \cdot s_{6}^{3}+K_{3} \cdot i \cdot s_{6}^{5} \\
& K_{10}=-\frac{8}{15} \cdot A G \\
& K_{11}=-\frac{16}{105} \cdot \frac{E I_{x}}{L^{2}} \\
& K_{12}=\frac{-K_{10}}{L} \\
& K_{13}=\frac{E I_{x}}{21 \cdot L^{3}} \\
& K_{14}=K_{10} \cdot K_{4}-K_{11} \cdot K_{4} \cdot s_{1}^{2}+K_{12} \cdot i \cdot s_{1}-K_{13} \cdot i \cdot s_{1}^{3} \\
& K_{15}=K_{10} \cdot K_{4}-K_{11} \cdot K_{5} \cdot s_{2}^{2}+K_{12} \cdot i \cdot s_{2}-K_{13} \cdot i \cdot s_{2}^{3} \\
& K_{16}=K_{10} \cdot K_{4}-K_{11} \cdot K_{6} \cdot s_{3}^{2}+K_{12} \cdot i \cdot s_{3}-K_{13} \cdot i \cdot s_{3}^{3} \\
& K_{17}=K_{10} \cdot K_{4}-K_{11} \cdot K_{7} \cdot s_{4}^{2}+K_{12} \cdot i \cdot s_{4}-K_{13} \cdot i \cdot s_{4}^{3} \\
& K_{18}=K_{10} \cdot K_{4}-K_{11} \cdot K_{8} \cdot s_{5}^{2}+K_{12} \cdot i \cdot s_{5}-K_{13} \cdot i \cdot s_{5}^{3} \\
& K_{19}=K_{10} \cdot K_{4}-K_{11} \cdot K_{9} \cdot s_{6}^{2}+K_{12} \cdot i \cdot s_{6}-K_{13} \cdot i \cdot s_{6}^{3} \\
& K_{20}=-K_{13} \cdot L \\
& K_{21}=-K_{11} \cdot L \\
& K_{22}=-K_{20} \cdot s_{1}^{2}+K_{21} \cdot K_{4} \cdot i \cdot s_{1} \\
& K_{23}=-K_{20} \cdot s_{2}^{2}+K_{21} \cdot K_{5} \cdot i \cdot s_{2} \\
& K_{24}=-K_{20} \cdot s_{3}^{2}+K_{21} \cdot K_{6} \cdot i \cdot s_{3} \\
& K_{25}=-K_{20} \cdot s_{4}^{2}+K_{21} \cdot K_{7} \cdot i \cdot s_{4}
\end{aligned}
$$




$$
\begin{aligned}
& K_{26}=-K_{20} \cdot s_{5}^{2}+K_{21} \cdot K_{8} \cdot i \cdot s_{5} \\
& K_{27}=-K_{20} \cdot s_{6}^{2}+K_{21} \cdot K_{9} \cdot i \cdot s_{6} \\
& K_{28}=\frac{16}{105} \cdot \frac{E I_{x}}{L^{2}} \\
& K_{29}=-\frac{68}{105} \cdot \frac{E I_{x}}{L} \\
& K_{30}=-K_{28} \cdot s_{1}^{2}+K_{29} \cdot K_{4} \cdot i \cdot s_{1} \\
& K_{31}=-K_{28} \cdot s_{2}^{2}+K_{29} \cdot K_{5} \cdot i \cdot s_{2} \\
& K_{32}=-K_{28} \cdot s_{3}^{2}+K_{29} \cdot K_{6} \cdot i \cdot s_{3} \\
& K_{33}=-K_{28} \cdot s_{4}^{2}+K_{29} \cdot K_{7} \cdot i \cdot s_{4} \\
& K_{34}=-K_{28} \cdot s_{5}^{2}+K_{29} \cdot K_{8} \cdot i \cdot s_{5} \\
& K_{35}=-K_{28} \cdot s_{6}^{2}+K_{29} \cdot K_{9} \cdot i \cdot s_{6}
\end{aligned}
$$

\section{References}

[1] S.P. Timoshenko, On the correction for shear of the differential equation for transverse vibrations of prismatic bars, Philosophical Magazine 41 (1921), 744-746.

[2] G.R. Cowper, The shear coefficient in Timoshenko's beam theory, Journal of Applied Mechanics 33 (1966), 335-340.

[3] A.V. Murthy, Vibration of short beams, AIAA 8 (1970), 34-38.

[4] J.J. Tuma and F.Y. Cheng, Theory and Problems of Dynamic Structural Analysis, Schaum's Outline Series, Mc Graw-Hill, New York, 1983.

[5] F. Gruttmann and W. Wagner, Shear coefficient factors in Timoshenko's beam theory for arbitrary shaped cross-section, Computational Mechanics 27 (2001), 199-207.

[6] S.M. Han, H. Benaroya and T. Wei, Dynamics of transversely vibrating beams using four engineering theories, Journal of Sound and Vibration 225 (1999), 935-988.

[7] W.B. Bickford, A consistent higher order beam theory, Development in Theoretical and Applied Mechanics 11 (1982), 37-150.

[8] M. Levinson, A new rectangular beam theory, Journal of Sound and Vibration 74 (1981), 81-87.

[9] P.R. Heyliger and J.N. Reddy, A higher-order beam finite element for bending and vibration problems, Journal of Sound and Vibration 126 (1988), 309-326.

[10] C.M. Wang, J.N. Reddy and K.H. Lee, Shear Deformable Beams and Plates: Relationships with Classical Solutions, Elsevier Science Ltd., The Netherlands, 2000.

[11] Y. Chen, On the vibration of beams or rods carrying a concentrated mass, Journal of Applied Mechanics 30 (1963), 310-311.

[12] C.H. Chang, Free vibration of a simply supported beam carrying a rigid mass at the middle, Journal of Sound and Vibration 237 (2000), 733-744.

[13] E.H. Dowell, On some general properties of combined dynamical systems, Transactions of the ASME 46 (1979), $206-209$.

[14] M. Gürgöze, A note on the vibrations of restrained beams and rods with point masses, Journal of Sound and Vibration 96 (1984), $461-468$.

[15] M. Gürgöze, On the vibration of restrained beams and rods with heavy masses, Journal of Sound and Vibration 100 (1985), $588-589$.

[16] M. Gürgöze, On the eigenfrequencies of a cantilever beam with attached tip mass and a spring-mass system, Journal of Sound and Vibration 190 (1996), 149-162.

[17] M. Gürgöze, On the alternative formulations of the frequency equation of a Bernoulli-Euler beam to which several spring-mass systems are attached in-span, Journal of Sound and Vibration 217 (1998), 585-595.

[18] H.Y. Lin and Y.C. Tsai, On the natural frequencies and mode shapes of a uniform multi-span beam carrying multiple point masses, Structural Engineering and Mechanics 21 (2005), 351-367.

[19] H.Y. Lin and Y.C. Tsai, On the natural frequencies and mode shapes of a multiple-step beam carrying a number of intermediate lumped masses and rotary inertias, Structural Engineering and Mechanics 22 (2006), 701-717.

[20] H.Y. Lin and Y.C. Tsai, Free vibration analysis of a uniform multi-span beam carrying multiple spring-mass systems, Journal of Sound and Vibration 302 (2007), 442-456.

[21] J.J. Wu, Free vibration analysis of a uniform beam carrying multiple spring-mass systems with masses of the springs considered, Structural Engineering and Mechanics 28 (2008), 659-676.

[22] W.H. Liu, J.R. Wuand and C.C. Huang, Free vibration of beams with elastically restrained edges and intermediate concentrated masses, Journal of Sound and Vibration 122 (1998), 193-207. 
[23] J.S. Wu and H.M. Chou, A new approach for determining the natural frequencies and mode shape of a uniform beam carrying any number of spring masses, Journal of Sound and Vibration 220 (1999), 451-468.

[24] S. Naguleswaran, Transverse vibrations of an Euler-Bernoulli uniform beam carrying several particles, International Journal of Mechanical Science 44 (2002), 2463-2478.

[25] S. Naguleswaran, Transverse vibration of an Euler-Bernoulli uniform beam on up o five resilient supports including ends, Journal of Sound and Vibration 261 (2003), 372-384.

[26] D. Zhou, Free vibration of multi-span Timoshenko beams using static Timoshenko beam functions, Journal of Sound and Vibration 241 (2001), 725-734.

[27] J.S. Wu and D.W. Chen, Free vibration analysis of a Timoshenko beam carrying multiple spring-mass systems by using the numerical assembly technique, International Journal of Numerical Methods in Engineering 50 (2001), 1039-1058.

[28] H.P. Lin and S.C. Chang, Free vibration analysis of multi-span beams with intermediate flexible constraints, Journal of Sound and Vibration 281 (2005), 155-169.

[29] J.R. Wang, T.L. Liu and D.W. Chen, Free vibration analysis of a Timoshenko beam carrying multiple spring-mass systems with the effects of shear deformation and rotary inertia, Structural Engineering and Mechanics 26 (2007), 1-14.

[30] Y. Yesilce and O. Demirdag, Effect of axial force on free vibration of Timoshenko multi-span beam carrying multiple spring-mass systems, International Journal of Mechanical Science 50 (2008), 995-1003.

[31] S. Kukla and B. Posiadala, Free vibrations of beams with elastically mounted masses, Journal of Sound and Vibration 175 (1994), 557-564.

[32] H. Su and J.R. Banerjee, Exact natural frequencies of structures consisting of two part beam-mass systems, Structural Engineering and Mechanics 19 (2005), 551-566. 

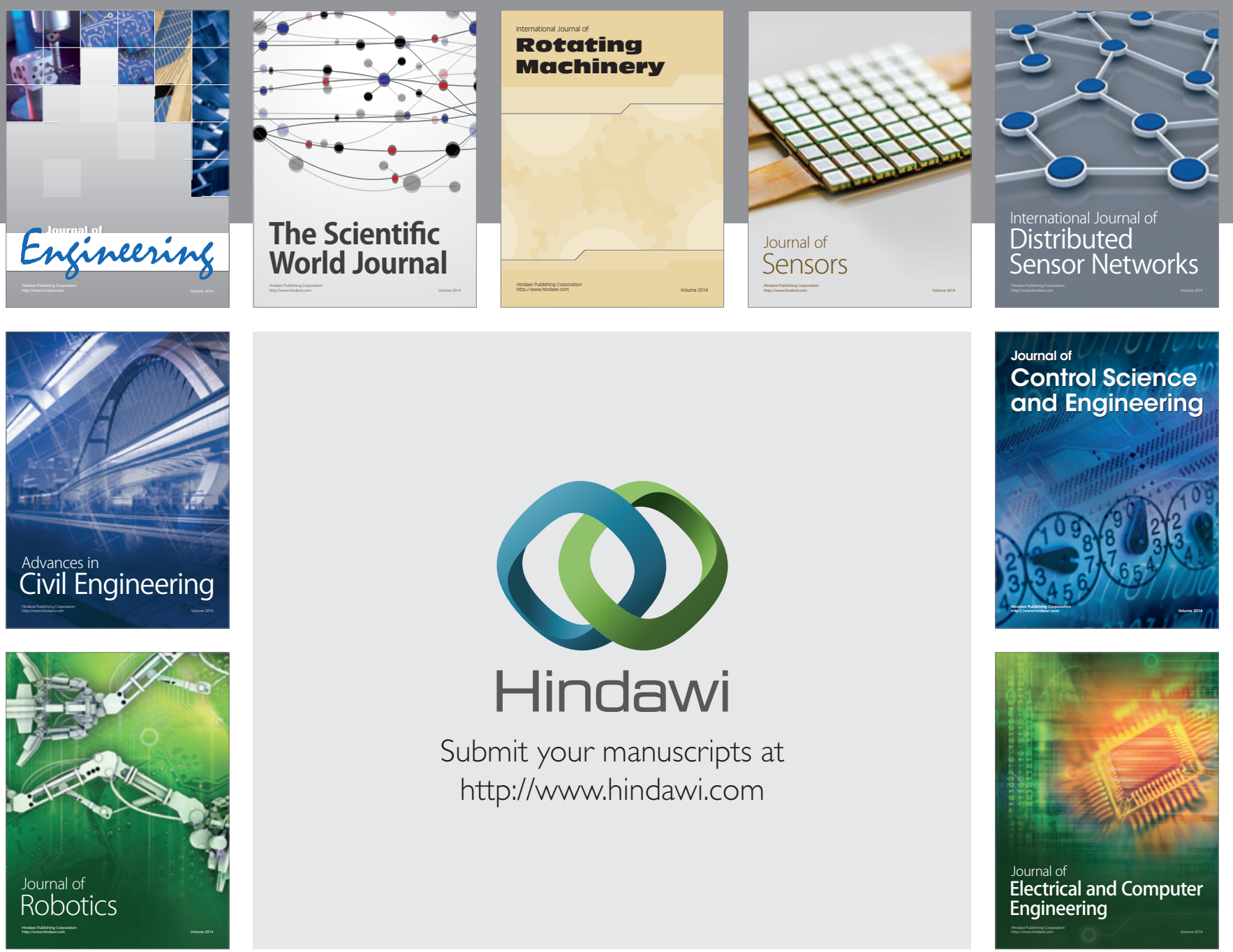

Submit your manuscripts at

http://www.hindawi.com
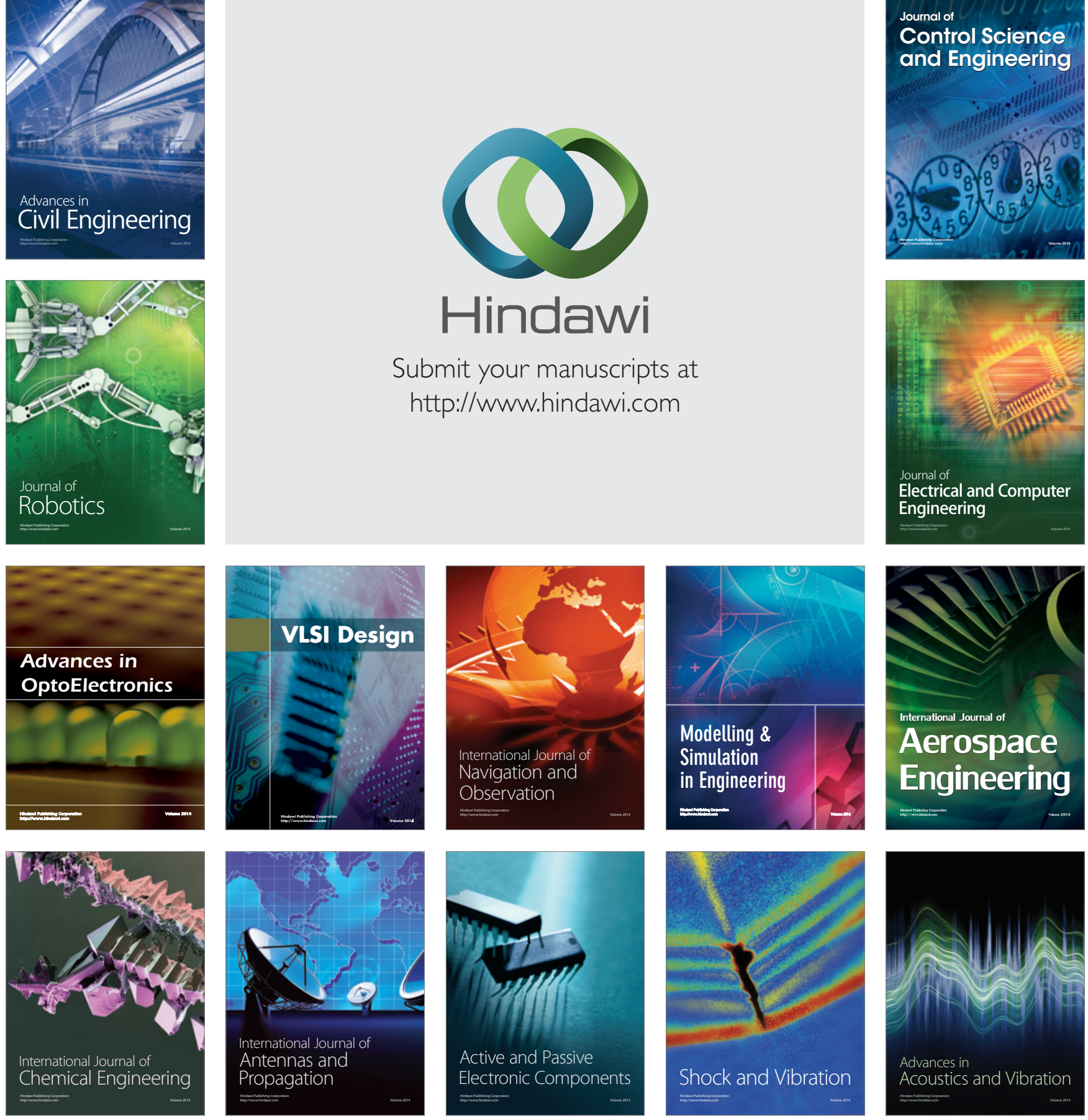